\title{
Dear Sir/Madame,
}

We would like to invite you to participate in the Project concerning improvement of HCV diagnosis in the primary health care. This questionnaire aims at collecting your experience, which will be analysed together with the test results in order to develop recommendations for provider-initiated testing offered by the primary health care doctors. The questionnaire is fully anonymous. Any identifying information collected will not be released and all the data will be used only for scientific purpose.

We ask you to provide accurate answers. This is the only way to assess the risks of hepatitis $C$.

Important: In case you have difficulties understanding some of the questions in the questionnaire please consult your doctor.

\section{General health}

1. Have you ever suffered from any of the diseases below:

$\begin{array}{ll}\text { haemophilia } & \square \text { Yes } \square \text { No } \\ \text { diabetes } & \square \text { Yes } \square \text { No } \\ \text { heart insufficiency / coronary heart disease } & \square \text { Yes } \square \text { No } \\ \text { astma/ COPD } & \square \text { Yes } \square \text { No } \\ \text { cancer } & \square \text { Yes } \square \text { No } \\ \text { kidney diseases } & \square \text { Yes } \square \text { No } \\ \text { complicated injuries } & \square \text { Yes } \square \text { No }\end{array}$

other chronic disease, due to which you were hospitalised 2 or more times:

$\square$ Yes, please list:

2. Have you ever been tested for hepatitis $\mathrm{C}(\mathrm{HCV})$ ?

$\square$ Yes

$\square$ No

$\square$ I don't know / I don't remember

\section{2a. If YES, what were the circumstances?}

$\square$ during hospitalisation (e.g. before surgical procedure)

$\square$ donating blood (after 1992)

$\square$ abnormal liver function tests, liver disease, abdominal pain

$\square$ partner/close contact diagnosed with the infection

$\square$ screening of risk group (e.g. people on dialysis, drug users, occupational exposure)

$\square$ own initiative (self-paid)

other circumstances, please list:

3. Have you ever received a positive test result of an HCV test?

$\square$ Yes

$\square$ No

$\square$ I don't know / I don't remember

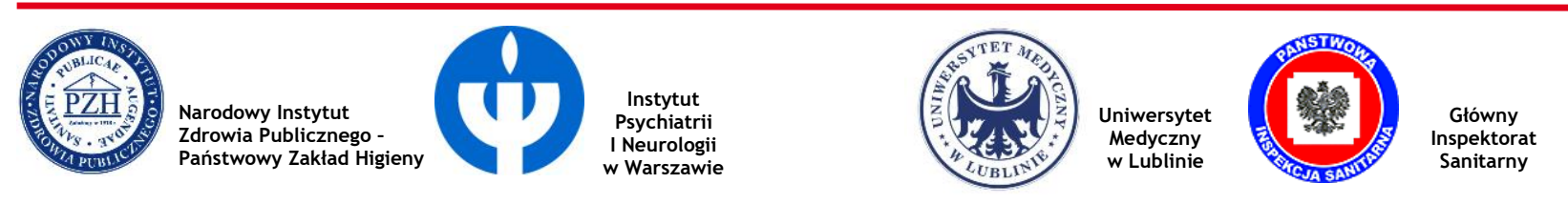




\section{$\checkmark$ SWISS \\ CONTRIBUTION}

4. Have you ever been infected or ill with the following diseases?

$\begin{array}{llll}\text { Hepatitis A } & \square \text { Yes } & \text { Year ........ } & \square \text { No } \square \text { I don't know } \\ \text { Hepatitis B } & \square \text { Yes } & \text { Year ....... } & \square \text { No } \square \text { I don't know } \\ \text { Hepatitis C } & \square \text { Yes } & \text { Year ....... } & \square \text { No } \square \text { I don't know } \\ \text { HIV } & \square \text { Yes } & \text { Year ....... } & \square \text { No } \square \text { I don't know }\end{array}$

\section{Medical exposures}

5. Have you ever received blood transfusion (including platelets, plasma, clotting factors, albumines, immunoglobulines)?

$\square$ No

$\square$ Yes, before 1992

$\square$ Yes, after 1992

$\square$ Yes, not remember date how many times: ......

how many times: .......

how many times: which blood element:

which blood element:

which blood element:

6. How many times have you been in the hospital (lifetime, excluding at birth)? (please include all admissions to hospital, including one-day stays and diagnostic stays)
$\square$ never
$\square 1-2$ times
$\square$ 3-4 times
$\square 5$ times and more

7. Have you ever had the following procedures performed on you:

Dental surgery

Endoscopy (e.g. gastroscopy, colonoscopy, bronchoscopy)

Small surgical procedures

$\square$ Yes $\square$ No
$\square$ Yes $\square$ No
$\square$ Yes $\square$ No

(e.g. wound sutures, abscess incision, nevus removal)

\section{Biopsy}

Caesarean section

Other procedures at delivery (e.g. episiotomy)

Major surgery (also otolaryngologic, cardiologic, orthopedic, gynecologic other that CC)

Other medical procedures, please list:

Dialysis $\square$ Yes $\square$ No

$\square$ Yes:how many times:... $\square$ No

$\square$ Yes:how many times:... $\square$ No

$\square$ Yes:how many times:... $\square$ No

$\square$ Yes $\square$ No

$\square$ Yes $\square$ No

8. During the last year did you have any injections (do not include blood collection)?

$\square$ Yes $\square$ No

8a. If YES please estimate the total number of injections during the last year in each category:

\begin{tabular}{|c|c|c|c|c|}
\hline & hospital & $\begin{array}{l}\text { In patient } \\
\text { clinic }\end{array}$ & At home & Other places \\
\hline $\begin{array}{l}\text { Medication by injection (e.g. } \\
\text { antibiotics, analgesics, steroids, } \\
\text { vitamins) }\end{array}$ & & & & \\
\hline Vaccination & & & & \\
\hline Anesthesia (also at dentist) & & & & \\
\hline Contrast, diagnostic tests & & & & \\
\hline i.v. line & & & & \\
\hline
\end{tabular}




\section{Non-medical exposures}

9. Have you ever had any of the following body modifications /non-medical procedures performed?

tattoo

$\square$ Yes, professional studio

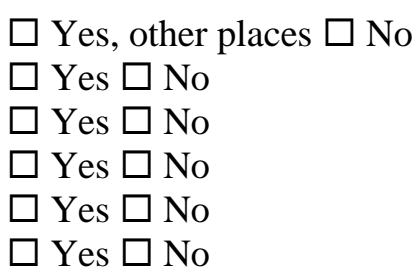

piercing

acupuncture

botox/mesotherapy

manicure/pedicure in beauty parlour

other cosmetic or non-conventional medicine procedures,

$\square$ Yes $\square$ No

please list:.

10. Have you ever been treated for alcohol addiction?

(in detox/ rehabilitation ward, AA groups)?

Yes $\square$ No

11. Have you ever used illegal drugs by injection or snorting?

$\square$ Yes, by injection $\quad \square$ Yes, snorting $\square$ Yes, snorting and injection

$\square$ No

12. Have you ever been detained for more than $\underline{3 \text { months }}$ (prison, correctional facility, detention centre)?

Yes $\square$ No

13. According to your best knowledge, is one of your current of past householders:

a.) Infected with HCV?

$\square$ Yes - what is your relationship with this person:

$\square$ No

b.) Injecting drug user?

$\square$ Yes - what is your relationship with this person:

$\square$ No

c.) Snorting drug user?

$\square$ Yes - what is your relationship with this person:

$\square$ No

14. According to your best knowledge, is one of your current of past sexual partners:
a.) Infected with $\mathrm{HCV}$
$\square$ Yes $\square$ No
b.) Injecting drugs user
$\square$ Yes $\square$ No
c.) Snorting drug user
$\square$ Yes $\square$ No 


\section{General information}

15. Sex: $\square$ Female $\square$ Male

\section{Education:}

$\square$ Elementary

$\square$ Occupational

$\square$ High school

$\square$ Post high-school

$\square$ Higher
16. Year of birth: $\left.\right|_{-}\|\|_{-}\|\|_{-} \mid$

\section{Residence:}

$\square$ city $>=100,000$ inhabitants
$\square$ city $50,000-99,999$
$\square$ city $20,000-49,999$
$\square$ city $<20,000$
$\square$ rural

19. Number of people living in your household:

20. Average monthly net income per household member during the past 12 months:
$\square<500$ PLN
$500-1000$ PLN
$1001-2500$ PLN
$2501-4000$ PLN
$>4000$ PLN

21. Have you ever worked in one of the following professions?:

Medical profession (direct contact with the patient) $\square$ Yes, what profession:

Cleaning/ removal of medical waste

$\square$ Yes $\square$ No

Work at diagnostic laboratory

$\square$ Yes $\square$ No

Fireman

Prison officer

Police, civil guard etc.

Work with people addicted to drugs or homeless

$\square$ Yes $\square$ No

$\square$ Yes $\square$ No

$\square$ Yes $\square$ No

$\square$ Yes $\square$ No

We would like to thank you for your time. 